Journal of Animal and Veterinary Advances 9 (15): 2030-2034, 2010

ISSN: $1680-5593$

(C) Medwell Journals, 2010

\title{
Detection of ESBLs and Antimicrobial Susceptibility of Escherichia coli isolated in Henan, China
}

\author{
${ }^{1,2}$ Chun-Hui Zhang, ${ }^{1}$ Yong-Lu Liu and ${ }^{1} \mathrm{~J}$ ian-Hua Wang \\ ${ }^{1}$ College of Animal Veterinary Medicine, Northwest A and F University, \\ Yangling, Shaanxi, 712100, People's Republic of China \\ ${ }^{2}$ Department of Pharmacy Engineering, Zhengzhou College of Animal Husbandry Engineering, \\ Henan, 450011, People's Republic of China
}

\begin{abstract}
A total of 51 pathogenic Escherichia coli isolates recovered from fowls and pigs were tested for production of Extended Spectrum $\beta$-Lactamases (ESBLs) by the Double Disc Synergy Test (DDST). For nine Enterobacteriaceaes tested for ESBLs by double-disk method we designed and synthetized two primer: TEM-1 and SHV-1 studied ESBLs gene type with amplified, sequenced and analyzed by PCR; the Minimal Inhibitory Concentrations ( $\mathrm{MICs}$ ) of Cetiofur, Ceftriaxone, Amoxicillin, Ampicillin to the nine Enterobacteriaceae producing ESBLs were carried out with two fold dilution method. The ESBLs producing bacteria amplified TEM type ESBLs fully but no SHV type ESBLs, the Minimal Inhibitory Concentrations (MICs) of Cetiofur, Ceftriaxone, Amoxicillin and Ampicillin were 10-80 $\mu \mathrm{g} \mathrm{mL}^{-1}, 40-80 \mu \mathrm{g} \mathrm{mL}^{-1},>128 \mu \mathrm{g} \mathrm{m}^{-1},>128 \mu \mathrm{gL}^{-1}$, respectively. This study reveals that $E$. coli isolated from chickens and swines in henan are resistant to the 3rd Cephalosporin Seriously and produce ESBL in large proportions.
\end{abstract}

Key words: ESBLs, resistance, detection, Escherichia coli, minimal inhibitory concentrations, antimicrobial susceptibility

\section{INTRODUCTION}

Enterobacteriaceae are major pathogens in animals as well as in humans. In recent years, the bacterial resistance to $\beta$-lactam antibiotics has risen dramatically (Katayama et al., 2004). Contributing to this has been the spread of extended spectrum $\beta$-Lactamases (ESBLs), enzymes that hydrolyze the expanded-spectrum Cephalosporins like cefotaxime and Ceftiofurand/or the monobactam (Davies et al., 2008).

ESBLs, a kind of $\beta$-lactamase were classified in group $2 \mathrm{~b}$ and subgroup $2 \mathrm{be}$ of the Bush-Jacoby-Medeiros classification schemeand were classified kind A in Ambler class (Bush et al., 1995).

The first $\beta$-lactamase originating from Enterobacteria was discovered and purified in 1964 it was marked as TEM-1 $\beta$-lactamase (Katsanis et al., 1994; Vinodkumar and Neelagund, 2006). The first ESBL which can hydrolyze the 3rd Cephalosporin detected in Klebsiella in German in 1985 (Kliebe et al., 1985). With the widespread use of 3rd generation cephalosporins, new ESBL has emerged continuously, there are more 300 kinds ESBLs now.
When bacteria were caused by ESBL producers, there is a delay in initiating an appropriate antibiotic treatment and high mortality rates (Rodriguez-Bano et al., 2006; Melzer and Perersen, 2007). $\beta$-Lactam antibiotics as Amoxicillin, Ceftiofur are frequently used to treat infections in veterinary clinic in China. In this study, the prevalence of ESBL production was evaluated and antimicrobial resistance in Enterobacteriaceae species in order to provide a guideline for the treatment about resistant bacterial infections in veterinary practices.

\section{MATERIALS AND METHODS}

Bacterial strains and culture conditions: From January 2006-September 2009, 51 non-duplicateg $E$. coli isolates were consecutively collected from different poultry and swine farms in Henan in China. The clinical isolates were identified as $E$. coli by using GNI cards on the VITEK- 32 automatic system (bioMerieux, France).

Escherichia coli ATCC 25922, C83907 and C83845 were used as the control strain were purchased from the China Institute of Veterinary Drug Control (Beijing, China). The strains were stored at $-4^{\circ} \mathrm{C}$ in nutritional broth until used.

Corresponding Author: Jian-Hua Wang, College of Animal Veterinary Medicine, Northwest A and F University, Yangling, Shaanxi, 712100, People's Republic of China 
Chemicals and enzymes: Taq DNA polymerase and dNTP were purchased from Takara Biotechnology Co. Ltd., (Dalian, P.R. China). GoldView nucleic acid dye and D2000 was purchased from Beijing Solarbio Science and Technology (Beijing. China). Cetiofur sodium and Ceftriaxone sodium-formulated as an intramuscular injection; Tazobactam sodium, Amoxicillin powder and Ampicillin powder were purchased from Qilu Pharmaceutical Co., Ltd (Shandong, China).

\section{Detection of ESBLs by Double Disc Synergy Test} (DDST): As described by Livermore and Brown (2001), bacteria were suspended equivalent to McFarland 0.5 turbidity standard in nutritional broth $\sim$ with Mueller-Hinton broth as test medium. After inoculating a Mueller-Hinton Agar plate, disks containing cefotaxime (CTX, $30 \mu \mathrm{g}$ each), ceftriaxone (CRO, $30 \mu \mathrm{g}$ each), ceftazidime (CAZ, $30 \mu \mathrm{g}$ each) were placed $25 \mathrm{~mm}$ (centre to centre) from an Amoxicillin-clavulanic acid disk ( 30 and $10 \mu \mathrm{g}$, respectively), incubated at $35^{\circ} \mathrm{C}$ over night. If there is synergy (combination of paper near the edge of the side appears to expand or enhance), determine the strains producing ESBLs. Escherichia coli ATCC 25922 was used as the quality control strain.

PCR detection of ESBLs genetype: The screening 9 clinic bacteria isolates further confirmed by Polymerase Chain Reactions (PCRs). As described previously (Tenover et al., 1995), the DNA extracted according to Plasmid Extraction Kit Manual. The DNA extraction was analyzed by electrophoresis on a $1.0 \%$ agarosegel and observed with UVI system (UVItec ST John's Innovation Centre, English).

PCRs for ESBL were conducted as the previously described data (Costa et al., 2009). The PCR primers for TEM: 5'-GAGTAT TCAACATTTCCGTGTCGC-3' (forward), 5'-TACCAATGCTTAATCAGTGAGGC-3' (reverse) and SHV: 5'-ATGCGTTATATTCGCCTGTG-3' (forward), 5'-TTAGCGTTGCCAGTGCTTGATC-3' (reverse).

The length of the expected amplified fragment was about 860 bp. The PCR amplification reactions were performed in a total volume of $50 \mu \mathrm{L}$. Each reaction mixture contained $5 \mu \mathrm{L}$ of $10 \times$ buffer, $4 \mu \mathrm{L}$ of dNTP, $1 \mu \mathrm{L}$ of each primer, $2 \mu \mathrm{L}$ DNA templates and $0.5 \mu \mathrm{L}$ of Taq DNA polymerase. PCR reaction was carried out in a Gene-Amp PCR system 2000 (Biometra, Gottinger, Germany) Programmed to perform. PCR reaction program: initial denaturation for $10 \mathrm{~min}$ at $94^{\circ} \mathrm{C}$ followed by 35 cycles consisting of denaturation at $94^{\circ} \mathrm{C}$ for $1 \mathrm{~min}$, annealing at $55^{\circ} \mathrm{C}$ for $1 \mathrm{~min}$ and an extension for $1.5 \mathrm{~min}$ at $72^{\circ} \mathrm{C}, 35$ cycles; the last extension step was extended to $8 \mathrm{~min}$ longer. PCR products were evaluated for the expected size and quantified on $1 \%$ agarosegels.
Sequencing: Both strands of PCR products were sequenced and identified kinds of ESBLs genes. Sequencing of the amplified products was performed with the ABIPRISM 377DNA automated sequencer (Takara Biotechnology Co. Ltd., Dalian, P.R. China). Sequences were analyzed and constructed the phylogenetic tree using the DNASTAR 7.10 (Integrated Sequence Analysis Software), software available on the website of the National Center for Biotechnology Information (accessed $26 \mathrm{Dec} 2009$ ).

Antimicrobial susceptibility testing: The Minimal Inhibitory Concentrations (MICs) of Amoxicillin, Ampicillin, Ceftriaxoneand Ceftiofur to the E.coli strains producing ESBLs were determined with the two-fold dilution method as previously described (Nakamura et al., 2009).

The concentration of the final inoculum was $1-5 \times 10^{5}$ cfu $\mathrm{mL}^{-1}$. The suspension were incubated aerobically at $35^{\circ} \mathrm{C}$ for $18-24 \mathrm{~h}$. The Minimum Inhibitory Concentrations (MICs) for each isolate was determined as the lowest dilution showing no visible bacteria growth clarify. The two quality control strains C83907 and C83845 were included in duplicate in each batch tested. Throughout the study, results were interpreted using the National Committee for Clinical Laboratory Standards (Clinical and Laboratory Standards Institute, 2006) for broth dilution and disc diffusion.

\section{RESULTS AND DISCUSSION}

Nine out of the $51 \mathrm{E}$. coli isolates from animal farms in Henan were found to be ESBLs-positive with DDST, (Fig. 1) resulting in an overall prevalence of $17.65 \%$. They were No 1 from chiaken in Kaifeng, No 3 from chicken in Xingyang, No 13 from chicken in Xinzheng, No 24 from chicken in Xinzheng, No. 25 from chicken in Yuanyang,

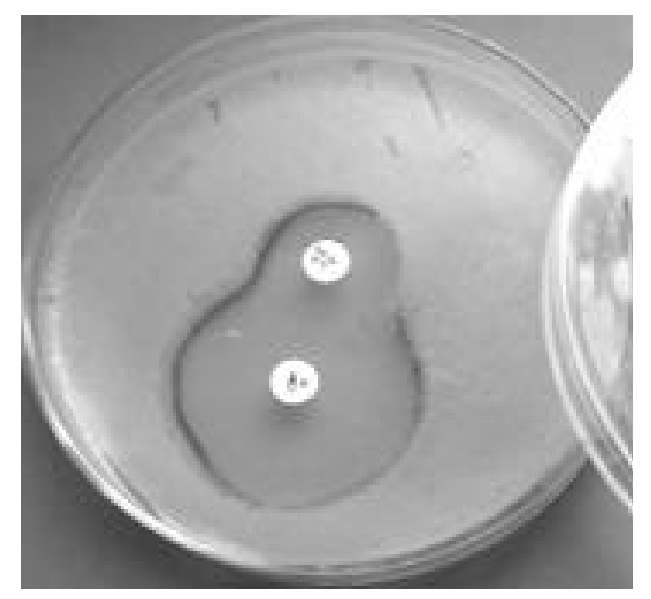

Fig. 1: Detection ESBLs by DDST 
Table 1: Results for detection of ESBLs by DDST

\begin{tabular}{lcc}
\hline & Bacteria & \\
\cline { 2 - 3 } Location of $E$. coli & Strain amount & Positive strain amount \\
\hline Kaifeng & 10 & 2 \\
Luohe & 6 & 1 \\
Yuanyang & 10 & 1 \\
Anyang & 7 & 1 \\
Xingyang & 12 & 2 \\
Xanzheng & 6 & 2 \\
Stand strains & 1 & 0 \\
\hline
\end{tabular}

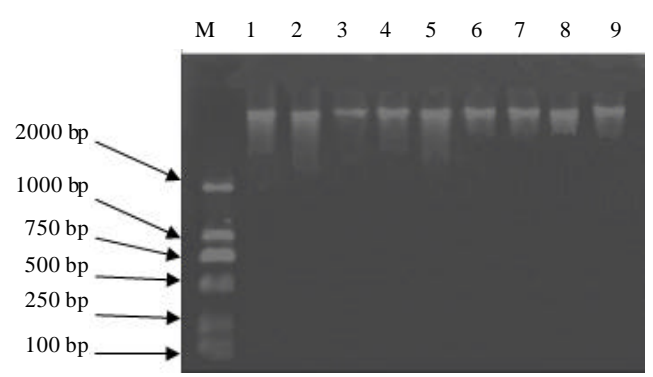

Fig. 2: Result of plasmid of Enterobacteriaceae ertracted of ESBLs, M. Marker, 1-9, T-1, T-2, T-3, T-4, T-5, $\mathrm{T}-6, \mathrm{~T}-7, \mathrm{~T}-8$ and $\mathrm{T}-9$

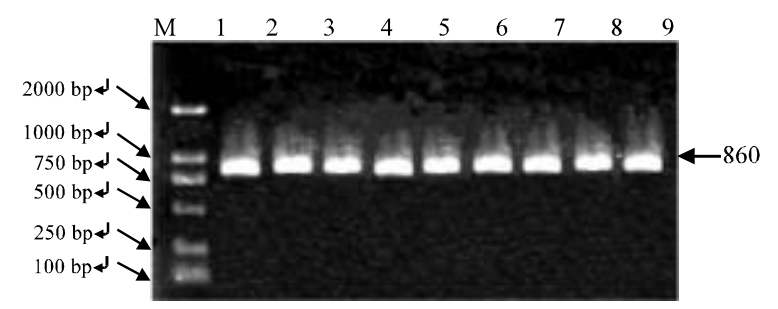

Fig. 3: PCR amplification of plasmid extracted from ESBLsM. DL2000 Marker, 1-9. PCR amplification of TEM primer of ESBLs for T-1, T-2, T-3, T-4, T-5, $\mathrm{T}-6, \mathrm{~T}-7, \mathrm{~T}-8$ and T-9

27 from chicken in Luohe, No 32 from chicken in Xingyang, No. 37 from swine in Anyang, No 43 from swine in Kaifeng. They were marked as T-1, T-2, T-3, T-4, $\mathrm{T}-5, \mathrm{~T}-6, \mathrm{~T}-7, \mathrm{~T}-8, \mathrm{~T}-9$, respectively, The results were shown in Table 1. Among the most relevant determinants of resistance emerging the worldwide in the Enterobacteriaceae. ESBLs are mainly associated with Enterobacteriaceae (Jacoby and Munoz-Price, 2005; Paterson and Bonomo, 2005). The similar proportion of ESBL-producing isolates in $E$. coli $(17.65 \%)$ has been reported in previous studies ( Ko et al., 2008).

Figure 2 shows that ESBLs resistant plasmids were extracted in all ESBL-positive bacteria. This results show that results by DDST was in agreement with PCR. For the method DDST was convenient, it was concluded that it fit in with the laboratory. This was in agreement with previous findings (Akujobi et al., 2008). According to

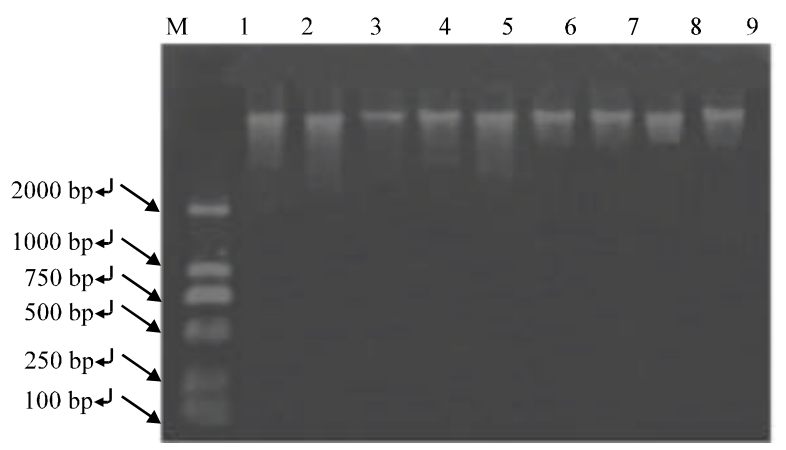

Fig. 4: PCR amplification of plasmid extracted from ESBLs M. DL2000 Marker, 1-9. PCR amplification of SHV primer of ESBLs, for T-1, T-2, T-3, T-4, T-5, T-6, $\mathrm{T}-7, \mathrm{~T}-8$ and T-9

PCR results (Fig. 3), the ESBLs producing bacteria by DDST, amplified TEM type ESBLs fully but no SHV type ESBLs (Fig. 4). And no bacteria both have TEM and SHV kind ESBL. In sequencing experiments, results show that those homologies among T-3 from Xinzheng, T-4 from Xinzheng, T-5 from Yuanyang were 99.9\%, the TEM ESBLs were same as each other in the two bacteria from Yuanyang.

Those homologies between T-1 from chickenin Kaifeng and T-9 from swine in Kaifeng were $100.0 \%$ but those homologies among T-6 from Luohe and this above ESBLs were 25.2-26.7\% only And those homologies among T-8 from Anyang and this above ESBLs were 23.0-26.5\% (Table 2 and Fig. 5).

Sequencing analysis results show that those homologies among ESBLs in the outskirts Zhengzhou were $31.9-00.0 \%$. But those homologies among T-6 bacteria from Luohe (a suburb of Zhengzhou)and this ESBL in the outskirt of Zhengzhou were $25.2-6.7 \%$ only those homologies among T-8 from Anyang and ESBLs in outskirt around Zhengzhou were $23.0-26.5 \%$ only. These result shows that ESBL producing was possible relation with the frequency of drug using.

The results for antimicrobial susceptibility are shown in Table 3. The MCs of Ceftiofur, Ceftriaxone, Amoxicillin, Ampicillin to the standard strains was $2,2,0.02$ and $0.2-0.4 \mu \mathrm{g} \mathrm{mL}^{-1}$, respectively.

In Table 3, the MIC of Ceftiofur to clinical E. coli is higher 80-400 times than the standard strains; the MIC of Ceftriaxone to clinical $E$. coli is higher 800-320 times than the standard strain; the $\mathrm{MLC}$ of Amoxicillin to clinical $E$. coli is higher 64 times than the standard strain. The MIC of Ampicillin to clinical E. coli is higher 64 times than the standard strain too. As we all known, every antimicrobial agent can select for resistant bacteria and 
Table 2: Percent identity about sequences of ESBLs

\begin{tabular}{lccccccccc}
\hline Bacteria & T-1 & T-2 & T-3 & T-4 & T-5 & T-6 & T-7 & T-8 & T-9 \\
\hline T-1 & - & 23.2 & 25.8 & 25.6 & 25.7 & 26.5 & 25.6 & 25.2 & 100.0 \\
T-2 & 350.0 & - & 23.1 & 23.1 & 23.0 & 23.0 & 31.9 & 25.2 & 23.2 \\
T-3 & 421.7 & 350.0 & - & 100.0 & 99.9 & 24.9 & 25.3 & 26.7 & 25.8 \\
T-4 & 350.0 & 350.0 & 0.0 & - & 99.9 & 25.1 & 25.1 & 26.6 & 25.6 \\
T-5 & 350.0 & 350.0 & 0.1 & 0.1 & - & 24.8 & 25.5 & 26.7 & 25.7 \\
T-6 & 299.1 & 350.0 & 350.0 & 350.0 & 350.0 & - & 23.8 & 26.7 & 26.5 \\
T-7 & 350.0 & 183.7 & 350.0 & 350.0 & 350.0 & 350.0 & - & 25.2 & 25.6 \\
T-8 & 350.0 & 448.4 & 298.5 & 301.0 & 298.5 & 305.3 & 350.0 & - & 25.2 \\
T-9 & 0.0 & 350.0 & 421.7 & 350.0 & 350.0 & 299.1 & 350.0 & 350.0 & - \\
\hline
\end{tabular}

Data above-means were percent identity, data below-means were divergence

Table 3: MIC of $\beta$-lactam antibiotics to Enterobacteriaceae producing ESBLs ug mL ${ }^{-1}$

\begin{tabular}{lrrrrrrrrrrr}
\hline Bacteria & $\mathrm{T}-1$ & $\mathrm{~T}-2$ & $\mathrm{~T}-3$ & $\mathrm{~T}-4$ & $\mathrm{~T}-5$ & $\mathrm{~T}-6$ & $\mathrm{~T}-7$ & $\mathrm{~T}-8$ & $\mathrm{~T}-9$ & $\mathrm{C}_{83907}$ & $\mathrm{C}_{83845}$ \\
\hline Amoxicillin & $>128$ & $>128$ & $>128$ & $>128$ & $>128$ & $>128$ & $>128$ & $>128$ & $>128$ & 2.00 & 2.00 \\
Ampicillin & $>128$ & $>128$ & $>128$ & $>128$ & $>128$ & $>128$ & $>128$ & $>128$ & $>128$ & 4.00 & 4.00 \\
Ceftriaxone & 128 & 64 & 80 & 64 & 40 & 32 & 64 & 80 & 64 & 0.04 & 0.04 \\
Cetiofur & 32 & 64 & 80 & 80 & 40 & 80 & 64 & 32 & 64 & 0.40 & 0.20 \\
\hline
\end{tabular}

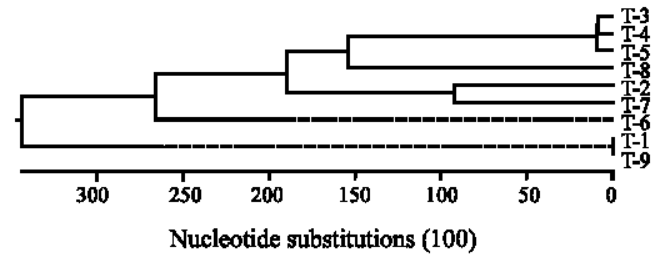

Fig. 5: Phylogenetic tree of TEM ESBLs1-9. Sequence of ESBLs for T-1, T-2, T-3, T-4, T-5, T-6, T-7, T-8 and T-9

hence the choice of the most effective antimicrobial agent(s) in the control of bacterial infections. Determination of valid data for the in vitro susceptibility of bacteria is an essential requirement to support the clinician's decision against or for a particular antimicrobial agent.

\section{CONCLUSION}

These result shows that ESBL producing was possible relation with the frequency of drug using. ESBLs-positive $E$. coli in Henan have not transmitted resistant gene through plasmids. The 3rd generation of cephalosporin has been resisted seriously in the veterinary clinic.

\section{ACKNOWLEDGEMENTS}

This research was done in the center Laboratory, Zhengzhou College of Animal Husbandry Engineering. This research was financially supported by the Key Science and Technology Program of Henan Province, China (072102130009) and the Natural Science Fund of Education Department of Henan Province, China (2006230004, 2009A230009).

\section{REFERENCES}

Akujobi, C.O., J.N. Ogbulie and C.S. Alisi, 2008. Occurrence of extended-spectrum b-lactamases in Escherichia coli isolated from piggery farms in Imo State, Nigeria. World J. Microbiol. Biotechnol., 24: $2167-2170$.

Bush, K., G.A. Jacoby and A.A. Medeiros, 1995. A functional classification scheme for $\beta$-lactamases and its correlation with molecular structure. Antimicrob. Agents Chemother., 39: 1211-1233.

Clinical and Laboratory Standards Institute, 2006. Performance Standards for Antimicrobial Susceptibility Testing Sixteenth Informational Supplement. Clinical and Laboratory Standards Institute, Wayne, PA., USA., ISBN: 1-56238-588-7.

Costa, D.L. P. Vinue, A.C. Poeta, M. Coelho and Y. Matos et al., 2009. Prevalence of extendedspectrum beta-lactamase-producing Escherichia coli isolates in faecal samples of broilers. Vet. Micro., 138: 339-344.

Davies, T.A., W. Shang, K. Bush and R.K. Flamm, 2008. Activity of doripenem and comparator b-lactams against US clinical isolates of Streptococcus pneumoniae with defined mutations in the penicillin-binding domains of pbpla, pbp2b and pbp2x. J. Antimicrob. Chemother., 61: $751-753$.

Jacoby, G.A. and L.S. Munoz-Price, 2005. The new $\beta$ lactamases. N. Engl. J. Med., 352: 380-391.

Katayama, H.Z. Y. Zhang and H.F. Chambers, 2004. PBP 2a mutations producing very-high-level resistance to beta-lactams. Antimicrob. Agents Chemother., 48: 453-459. 
Katsanis, P.G. J. Spargo, M.J. Ferraro, L. Sutton and G. Jacoby, 1994. Detection of Klebsiella pneumoniae and Escherichia coli strains producing extendedspectrum $\beta$-lactamases. J. Clin. Microbiol., 32: 691-696.

Kliebe, C., B.A. Nies, S.F. Meyer, R.M. TolxdorffNeutzling and B. Wiedeman, 1985. Evolution of plasmid-coded resistance to broad-spectrum cephalosporin. Antimicrob. Agents Chemother., 28: 302-307.

Ko, K.S., M.Y. Lee, J.H. Song, H. Lee and D.S. Jung et al., 2008. Prevalence and characterization of extended-spectrum $\quad \beta$-lactamase-producing Enterobacteriaceae isolated in Korean hospitals. Diagnn. Microbiol. Infect. Dis., 61: 453-459.

Livermore, D.M. and D.F.J. Brown, 2001. Detection of $\beta$ lactamase-mediated resistance. J. Antimicrob. Chemother., 48: 59-64.

Melzer, M. and I. Perersen, 2007. Mortality following bacteraemic infection caused by extended spectrum $\beta$-lactamase (ESBL) producing $E$. coli compared to non-ESBL producing $E$. coli compared to non-ESBL producing $E$. coli. J. Infect., 55: 254-259.
Nakamura, T., C. Shimizu, K. Hirakawa, S. Inui and K. Okuda et al., 2009. Antibacterial activity of quinolones against various clinically isolated strains and evaluation of efficacy based on the pharmacokinetics/pharmacodynamics theory. Jap. J. Antibiot., 62: 194-202.

Paterson, D.L. and R.A. Bonomo, 2005. Extendedspectrum $\beta$-lactamases: A clinical update. Clin. Microbiol. Rev., 18: 657-686.

Rodriguez-Bano, J., M.D. Navarro, L. Romero, M.A. Muniain and M.D. Cueto et al., 2006. Bacteremia due to extended-spectrum beta-lactamase-producing Escherichia coli in the CTX-Mera: A new clinical challenge. Clin. Infect. Dis., 43: 1407-1414.

Tenover, F.C., R.D. Arbeit, R.V. Goering, P.A. Mickelsen, B.E. Murray, D.H. Persing and B. Swaminathan, 1995. Interpreting chromosomal DNA restriction patterns produced by pulsed-field electrophoresis: Criteria for bacterial strain typing. J. Clin. Microbiol., 33: 2233-2239.

Vinodkumar, C.S. and Y.F. Neelagund, 2006. Emergence of extended spectrum beta lactamase mediated resistance in neonatal septicemia. Indian J. Pathol. Microbiol., 49: 616-619. 